\title{
Theoretical and experimental study of ion-exchange process on zeolites from 5-1 structural group
}

\author{
M. Król ${ }^{1} \cdot$ W. Mozgawa ${ }^{1} \cdot$ W. Jastrzębski ${ }^{1}$
}

Published online: 13 August 2015

(c) The Author(s) 2015. This article is published with open access at Springerlink.com

\begin{abstract}
In this work the DFT calculations were carried out by means of Gaussian09 program for chosen model cluster-mor Composite Building Unit of zeolites structures. Vibrational frequencies computations and infrared spectra were obtained subsequently for above model. The results of calculations have been compared with IR (MIR and FIR range) spectroscopic studies of zeolites belonging to 5-1 structural group (mordenite and ferrierite) after heavy metal cations $\left(\mathrm{Ag}^{+}, \mathrm{Zn}^{2+}, \mathrm{Cd}^{2+}, \mathrm{Pb}^{2+}\right.$, and $\left.\mathrm{Cr}^{3+}\right)$ immobilization. Changes in intensities and positions of the bands corresponding to the characteristic ring and $\mathrm{Me}-\mathrm{O}$ vibrations have been observed. These rings occur in pseudomolecular complexes (built of $\left[\mathrm{SiO}_{4}\right]$ and $\left[\mathrm{AlO}_{4}\right]$ tetrahedra) which constitute the secondary building units and form zeolite framework. Based on the results of DFT method calculations, normal vibrations of the 5-1 unit terminated by different cations $\left(\mathrm{Na}^{+}, \mathrm{K}^{+}\right)$have been visualized. Obtained results have been applied for interpretation of experimental spectra of selected zeolites. The most significant changes have been determined in the region of pseudolattice vibrations $\left(800-500 \mathrm{~cm}^{-1}\right.$ ), as well as $\mathrm{Me}-\mathrm{O}$ vibrations (below $400 \mathrm{~cm}^{-1}$ ). It was proven those cations' sorption causes changes in the experimental spectra of zeolites. Sorption has been conducted on monoionic forms of mordenite and ferrierite. Based on the results of the sorption/desorption
\end{abstract}

Electronic supplementary material The online version of this article (doi:10.1007/s10934-015-0050-6) contains supplementary material, which is available to authorized users.

\section{Król}

mkrol@agh.edu.pl

1 Faculty of Materials Science and Ceramic, AGH University of Science and Technology, 30 Mickiewicza Av., 30-059 Kraków, Poland experiments, the proportion of ion-exchange to chemisorption in the process and the effective cation exchange capacity of the individual samples have been estimated. Results of AAS studies have been compared with those obtained by vibrational (IR and Raman) spectroscopy. Changes in intensities and positions of the bands corresponding to the characteristic ring vibrations, due to the immobilization of heavy metal ions, have been observed.

Keywords FT-IR spectra - Zeolite - Mordenite . Ferrierite $\cdot$ Sorption

\section{Introduction}

Due to the specific chemical and physicochemical properties resulting in very wide use in many areas of chemistry and chemical technology, structures of zeolites are the subject of numerous studies (including the use of vibrational spectroscopy), which aim is to link the macroscopic characteristics of zeolite materials with their internal build.

As mentioned, zeolites have a lot of applications, among which the most important is their use in the process of heavy metal cations immobilization. Cations can be immobilized on aluminosilicates by two mechanisms: ion-exchange and chemisorption [1]. Ion-exchange process leads to creation of new kind of bonds and small deformation of the initial zeolite structure, so it is possible to observe changes in the IR-spectra of zeolites which are result of heavy metal cations immobilization. Previous studies [2,3] showed that incorporation of cations into the zeolite structure results mainly in changes of the intensity of the bands associated with ring-opening (RO type) vibrations as well as modification of bands due to Me-O vibrations in FIR spectra (below $400 \mathrm{~cm}^{-1}$ ). 
Interpretation of infrared spectra of zeolites often is difficult and in such situations computational methods can be helpful. However, regardless of the method used to interpret the spectra of zeolites the basic problem is the choice of a fragment of the crystal structure. First, it cannot be a single tetrahedron, because such units are present in all the structures of silicates and aluminosilicates, not only in zeolites. Unit cell is also not useful, because its size can be up to hundreds of atoms [4]. While, systems of tetrahedra forming so-called Secondary Building Units (SBU), such as single and double rings, can be such model units. For such fragments of framework, theoretical models in the form of isolated pseudomolecules terminated by different cations can be built [5]

Mordenite's structure is a framework containing chains of five-membered rings of linked silicate and aluminate. These building units form structure with straight 12-membered ring channels $(6.7 \times 7.0 \AA)$ (parallel to [001]) and crossed 8-membered ring channels $(2.9 \times 5.7 \AA)$ (parallel to [010]). Similarly the ferrierites structure is characterized by 2-dimensional channel system, which are formed by 10 $(3.4 \times 5.5 \AA)$ and 8-membered $(3.4 \times 4.8 \AA)$ ring channels $[6,7]$. Isolated rings with such a large diameter are not sufficiently stable for the calculation and during geometry optimization assume a linear conformation. On the other hand, the SBU of this group of minerals has the lowest possible symmetry. In addition, in the case of this unit, due to a different number of tetrahedral atoms in 5-membered ring and distinct ring deformation in 5-1 unit it is hard to talk about typical RO vibrations. However, based on ours previous works [8], presence of vibrations that are characteristic for all SBUs can be expected, such as it was in the case of 4-4-1 units in relation to the structure of clinoptilolite. On the other hand, the cations depending on the location in the zeolite framework occupy different crystallographic sites, and thus show characteristic vibrational modes in the FIR spectra [9]. But cations position determination based on the IR spectrum is difficult, especially in the case of high-silica zeolites, for which the non-tetrahedral cation concentration is low.

The aim of this work is to examine the usefulness of quantum-mechanical calculated methods coupled with founded model for the interpretation of experimental spectra as well as the influence of non-tetrahedral cations on shape of their envelopes. Additionally, the results of heavy metal cations $\left(\mathrm{Cu}^{2+}, \mathrm{Zn}^{2+}, \mathrm{Cd}^{2+}, \mathrm{Pb}^{2+}\right.$ and $\left.\mathrm{Cr}^{3+}\right)$ sorption studies on mordenite and ferrierite are described.

\section{Experimental}

\subsection{Computational details}

Initial atomic positions taken for calculations were cut from FER structure. Selected cluster (Fig. 1), which corresponds to the mor Composite Building Unit (according to [7]), were terminated by $\mathrm{Na}^{+}$or $\mathrm{K}^{+}$nontetrahedral cations (it is built with 36 atoms and the resulting charge was zero). All calculations were carried out by means of Gaussian09 program within DFT formalism using B98 XC hybrid potential and LanL2DZ basis set. The geometries of selected model clusters were optimized in order to find energy minimum on potential energy hypersurface. For each cluster with optimized geometry the hessian matrix was calculated and eigenvalue problem solved leading to a set of normal modes of vibrations and respective frequencies. Additionally, for every cluster, both the IR and Raman intensities were calculated as well as both spectra simulated. Calculated frequencies were remained unscaled. Infrared spectra were constructed assuming a $30 \mathrm{~cm}^{-1}$ full width at half maximum (FWHM) of calculated bands $\left(15 \mathrm{~cm}^{-1}\right.$ for Raman). All eigenmodes were visualized (animated) assuming simple sinusoidal motion of atoms along the trajectory determined by a given eigenmode values. Calculated trajectory of atomic oscillation around equilibrium position consisted of 15 frames of animation and was visualized by Accelrys Discovery Studio Visualizer.

Based on results obtained by Gaussian, the vibrational energy distribution (VED) was calculated. From the formulas for potential energy of a point hooked on the spring $\left(E_{p}=k x^{2} / 2\right)$ and the frequency of its vibration $(\omega=(k)$ $m)^{1 / 2}$ ) new formula for potential energy can be derived: $E_{p}=1 / 2 m x^{2} \omega^{2}$. Following the assumption that the deflection is a maximum (calculated amplitude), it will be an expression for the total energy. The frequency in the formula is a constant (in mode all of the atoms have the same frequency), so total energy of this material point is proportional to the expression $m A^{2}$, where $m$-mass of the atom and $A-$ deflection of this atom from the equilibrium

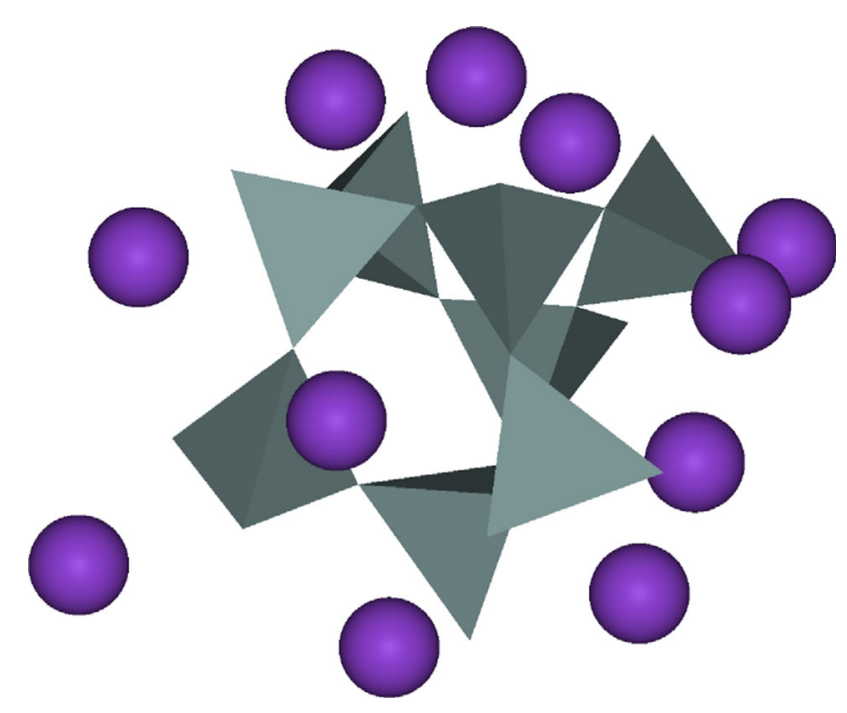

Fig. 1 Cluster for calculations 
position, calculated by Gaussian. Based on above assumption, for VED calculations the fallowing procedure was used. Expression $m A^{2}$ was calculated for each mode of normal for every atom. Each type of atom has been separately summed and calculated sums to $100 \%$ have been standardized. Each normal mod using the three types of atoms with the largest proportions have been described.

\subsection{Experimental details}

Calculated spectra were used in interpretation of the experimental ones. Sodium forms of mordenite and ferrierite (both from 5-1 structural group), available in commercial offer of the Zeolyst International, were used as the reference samples. The phase composition of the samples was confirmed by X-Ray diffraction study (Fig. 2). X-ray structural analyses were performed using Philips X-ray difractometer $\mathrm{X}^{\prime}$ Pert system $\left(\mathrm{Cu} \mathrm{K}_{\alpha}\right.$ radiation). Same additional information about starting materials, such as $\mathrm{Si}$ / $\mathrm{Al}$ ratio, amount of $\mathrm{Na}_{2} \mathrm{O}$ or surface area, is presented in Table 1.

Sorption of heavy metals was also carried out to test the effect of the presence of extra-framework cations on the envelope of the spectra. Selected cations were introduced into mordenite (MOR) and ferrierite (FER) structure from aqueous solutions of nitrates: $\mathrm{AgNO}_{3}, \mathrm{Zn}\left(\mathrm{NO}_{3}\right)_{2} \cdot 6 \mathrm{H}_{2} \mathrm{O}$, $\mathrm{Cd}\left(\mathrm{NO}_{3}\right)_{2} \cdot 4 \mathrm{H}_{2} \mathrm{O}, \mathrm{Pb}\left(\mathrm{NO}_{3}\right)_{2}$ and $\mathrm{Cr}\left(\mathrm{NO}_{3}\right)_{3} \cdot 9 \mathrm{H}_{2} \mathrm{O}(P O C H)$ respectively. The starting concentration of the metals was in the range $1 \times 10^{-3}$ to $20 \times 10^{-3} \mathrm{~mol} / \mathrm{dm}^{3}$. A suspension of the zeolite in water $\left(20 \mathrm{~g} / \mathrm{dm}^{3}\right)$ was shaken with the appropriate metal salt solution for $24 \mathrm{~h}$ at $25^{\circ} \mathrm{C}$ and centrifuged. All the sorption experiments were done in triplicates for each ion concentration. Atomic absorption spectroscopy (Philips PU-9100×) was used to determine the concentration of metal cations in the solutions before and after the sorption experiments. $\mathrm{pH}$ was controlled.

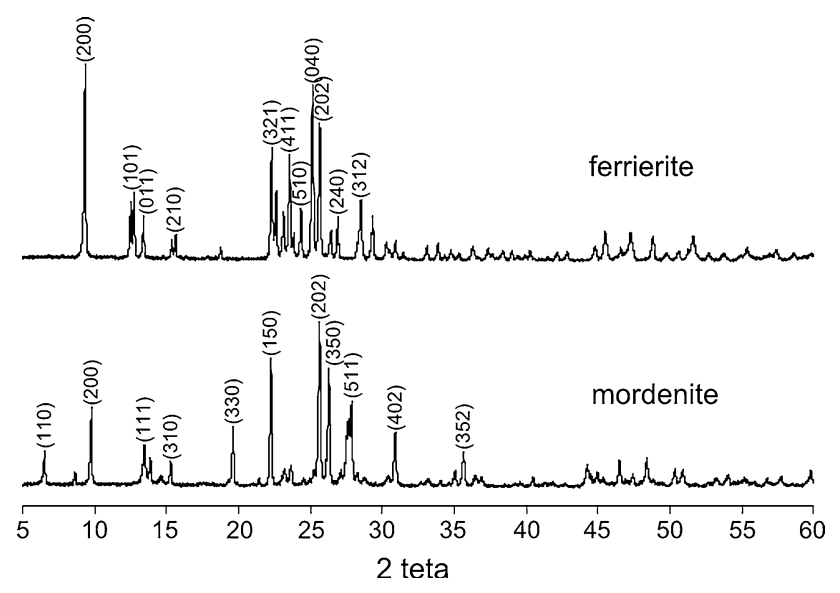

Fig. 2 XRD patterns of starting mordenite and ferrierite
After the ion-exchange process the samples were triply washed with distilled water. To desorb the exchanged metals, the mineral samples were flooded with $1 \mathrm{M} \mathrm{NaCl}$ or $\mathrm{CH}_{3} \mathrm{COONH}_{4}$ aqueous solution and the process was carried out similarly as in the case of sorption. Samples selected for spectroscopic studies were dried at $80^{\circ} \mathrm{C}$ for several days.

Results of AAS studies have been compared with those obtained by FT-IR spectroscopy, from which the influence of the sorbed cations on zeolite' structure has been determined. Infrared spectra were measured on a Bruker VERTEX 70v vacuum spectrometer. They were collected in the mid and far infrared region, after 256 scans at $2 \mathrm{~cm}^{-1}$ resolution. Samples were prepared using the standard $\mathrm{KBr}$ (Merck) and polyethylene (Merck) pellets methods for MIR and FIR spectra, respectively.

\section{Results and discussion}

The computed spectra carried out by DFT method are useful for interpretation of experimental once. It is possible to assign the bands to individual normal modes (Fig. 3). Figure 3 compares calculated IR spectrum of selected model cluster (Na-form of mor unit) with experimental spectra of the zeolites from 5-1 structural group (mordenite and ferrierite). The bands have been identified based on visualization of individual normal vibrations. Computed spectrum shows great similarity to the experimental spectra as to quantity and intensity groups of bands. This similarity allows moving the interpretation of the calculated spectra on the experimental.

The bands at 1067 and $1022 \mathrm{~cm}^{-1}$ in the spectrum of Na-mor unit correspond to the asymmetric stretching vibrations of $\mathrm{Si}-\mathrm{O}(\mathrm{Si})$ bridges and, as for most silicates, can be found as the most intense bands in the experimental spectra. It is worth noting that the analyzed structures are classified as highly siliceous zeolites (in this case $\mathrm{Si} / \mathrm{Al}$ ratio is 26 for modrenite and 40 for ferrierite), hence almost total absence of bands in the spectra derived from $\mathrm{Si}-\mathrm{O}-\mathrm{Al}$ bridges. Next group of bands in the computed spectrum (i.e. intensive maxima at 943, 910 and $815 \mathrm{~cm}^{-1}$ ) are assigned to vibrations of bonds associated with the presence of terminal oxygen $\left(\mathrm{Si}-\mathrm{O}^{-}\right)$and cannot be found in the spectra of real structures. Other groups of bands with lower intensities correspond to symmetric stretching (at 682 and $586 \mathrm{~cm}^{-1}$ in theoretical spectrum) and bending vibrations (at 519,425 and $359 \mathrm{~cm}^{-1}$ ). The above results show that even in the case of simple cluster model, the obtained results agree well with related literature [10, 11] and thus allow good description of experimental data.

The bands correspond to the so-called pseudo-lattice vibrations of larger parts of the structure can be divided. 
Table 1 Physicochemical parameters of starting zeolites

\begin{tabular}{lllll}
\hline Zeolite & $\mathrm{Si} / \mathrm{Al}$ ratio & Nominal cation form & $\mathrm{Na}_{2} \mathrm{O}(\mathrm{wt} \%)$ & Surface area $\left(\mathrm{m}^{2} / \mathrm{g}\right)$ \\
\hline Mordenite & 7.5 & Sodium & 6.5 & 425 \\
Ferrierite & 10 & Ammonium & 0.05 & 400 \\
\hline
\end{tabular}

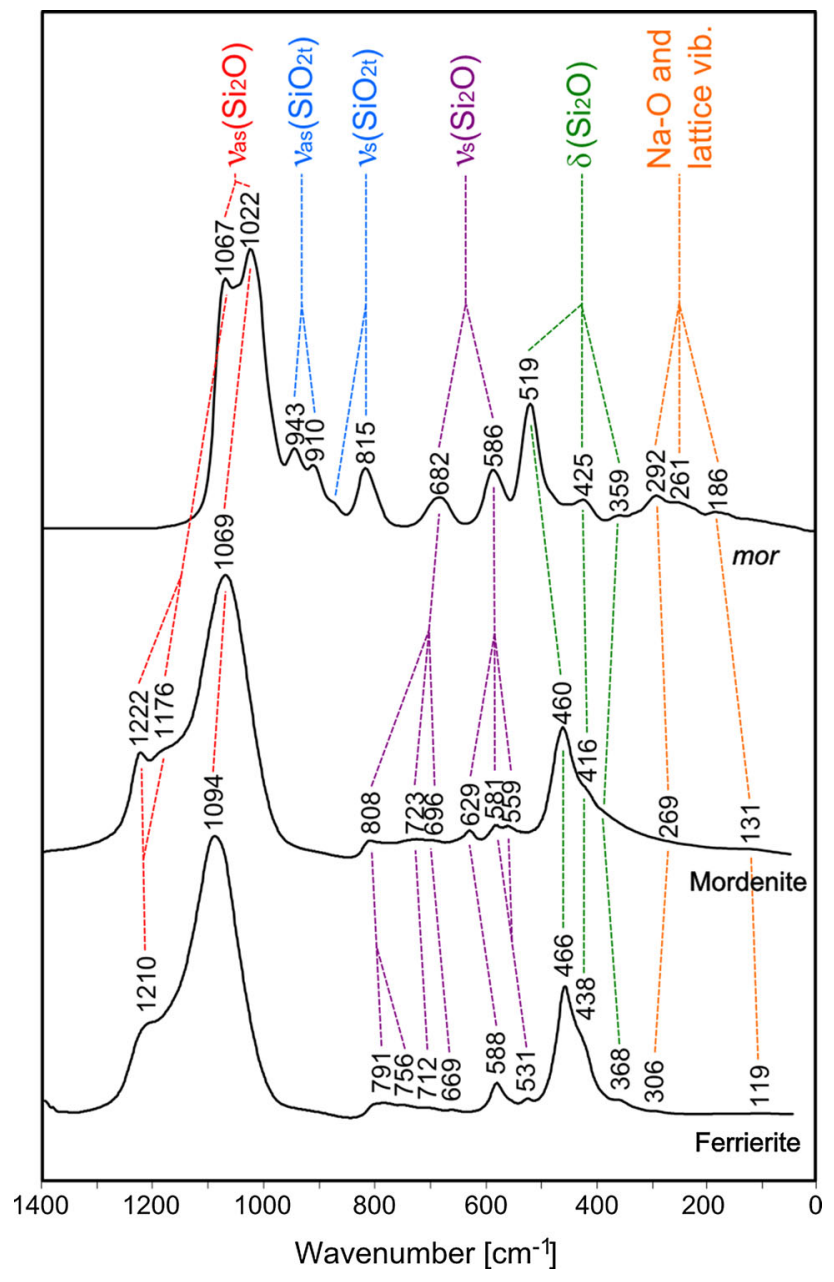

Fig. 3 Assignments of bands for calculated spectra of Na-form of mor unit and experimental spectrum of mordenite and ferrierite

Mor units occurring both in mordenite and ferrierite structures contain deformed five-membered rings of silicooxygen tetrahedra (Fig. 1). In the IR spectra, characteristic bands originating from "ring-like" vibrations of this unit can be identified (Figs. 4, 5). Ideal ring vibrations cause simultaneous and radial (with respect to the ring centre) displacements of atoms from their starting positions without changing the ring symmetry $[8,12]$. These vibrations can be both, stretching and bending in nature. As mentioned, in the case of analyzed unit, due to a different number of tetrahedral atoms in 5-membered ring and distinct ring deformation it is hard to talk about typical RO vibrations, however, the characteristic vibrations, which to a large extent fulfilled the criteria of ring vibrations $[8,12]$, of the whole unit can be identified at 524 (Fig. 4a) and $560 \mathrm{~cm}^{-1}$ (Fig. 5a) respectively for $\mathrm{Na}$ - and $\mathrm{K}$-form of model cluster. These vibrations were not implemented within individual rings, but rather in whole unit, so-also with some approximation - can be considered that they are characteristic for PO vibration typical for such units as D4R or D6R [2].

The presence of ring-like vibrations in is can be also confirmed by analysis of the computed Raman spectra (Fig. 6). For example, in the Raman spectrum of Na-mor, the bands derived from the characteristic vibrations, at 600 and $580 \mathrm{~cm}^{-1}$, have an increased intensity. On the other hand, for units with a high degree of deformation, several characteristic bands can be identified in the Raman spectrum, while only one intense band connected with fullsymmetrical breathing vibration (PO type) is presented in the spectra of symmetric units such as D4R or D6R. Similar relationships have been demonstrated in the work [8] for the 4-4-1 units.

One of the results of the carried out calculations is the information about the vibrational energy distribution (VED). It has been assumed that the characteristic vibrations of the mor unit are normal vibrations, related primarily to the symmetric stretching (or less bending) vibrations of $\mathrm{Si}-\mathrm{O}(\mathrm{Si})$, so during the implementation of these vibrations, silica atoms and/or bridging oxygen atoms the most move from the equilibrium sites. Therefore, in order to recognize characteristic ring-like vibrations, only those in which the percentage of energy vibration of the $\mathrm{Si}$ and bridging $\mathrm{O}$ atoms is the greatest should be taken into account. Characteristic vibrations of the $\mathrm{Me}-\mathrm{O}$ type (Figs. 4b, 5b), present in the FIR range, have been identified in a similar manner. In this case, the maximum share of vibration energy of non-tetrahedral cations, i.e. $\mathrm{Na}^{+}$and $\mathrm{K}^{+}$, was taken as major criterion.

It is expected, that metal cations sorption will affect the characteristic bands corresponding to the vibrations of mor structural units. Comparison of computed IR and Raman spectra of analyzed unit terminated by $\mathrm{Na}^{+}$and $\mathrm{K}^{+}$ions was shown in Fig. 6. Terminal ions differing in the atomic mass cause a considerable change in the frequency of vibrations characteristic for a given unit. It can be noticed that $\mathrm{Na}^{+} \rightarrow \mathrm{K}^{+}$cation exchange has generally resulted in band shift slightly towards lower wave numbers. The decrease in intensity is also visible in the spectra (Fig. 6) but they are not so significant. In contrast, in the Raman spectra clear decrease in the intensity of bands in the 
Fig. 4 Sample visualization of vibrations of mor unit, terminated by $\mathrm{Na}^{+}$cations, occurring at 524 (a) and $124 \mathrm{~cm}^{-1}$ (b)

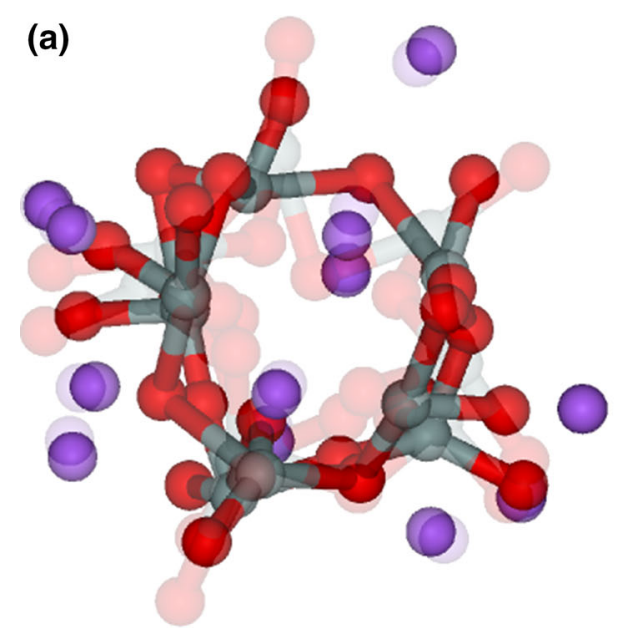

(a)

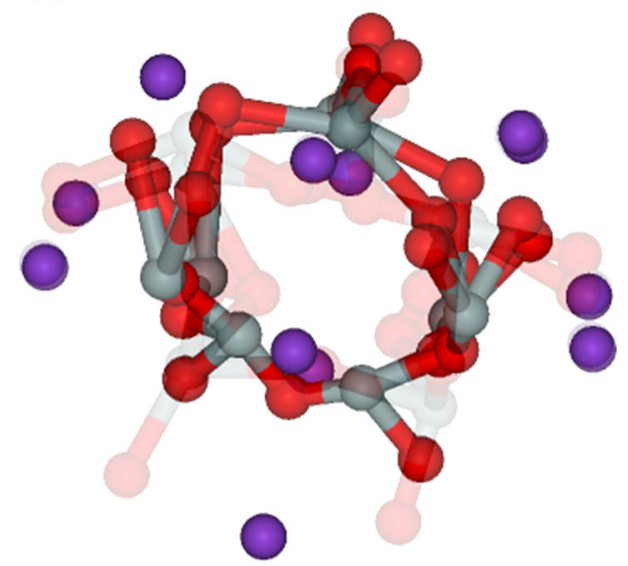

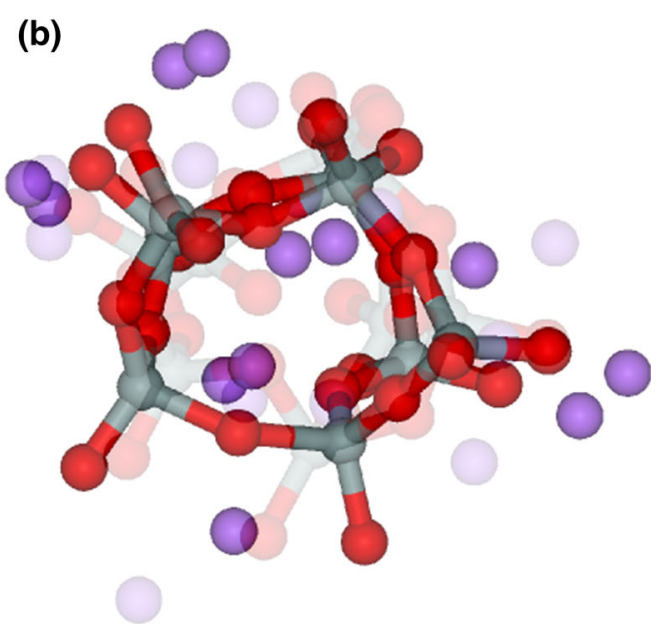

(b)

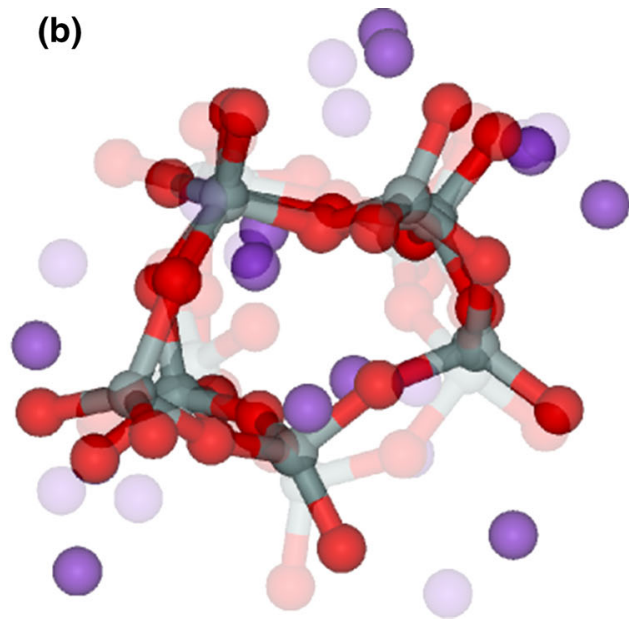

Fig. 5 Sample visualization of vibrations of mor unit, terminated by $\mathrm{K}^{+}$cations, occurring at 560 (a) and $111 \mathrm{~cm}^{-1}$ (b) pseudolattice vibration range is evident, which suggest, that ion-exchange could influence the deformation degree of the initial structure.

In real structures SBUs are more rigid due to the incorporation into a spatial framework of tetrahedra (single unit has more degrees of freedom than the whole structure). Is expected, that interpretation of vibrational spectra based on calculated for periodic models will be more clear, unambiguous and better matching to the experimental data, so calculations of this type are planned (e.g. by the use of Crystal). Additionally, presented model is useful mainly in interpretation of MIR spectra but they are not sufficient for interpretation of the whole FIR spectrum, due to the lack of proper description of lattice vibration. Therefore, taking into account the influence of the translational symmetry of crystal lattice could be theoretically studied for more accurate characterization of this range of the spectra. However, it should be noted that such calculations are extremely time-consuming due to the high unit cell size (up to several thousands of atoms).
The spectra calculated for chosen model were used in detailed analysis and interpretation of the experimental ones, collected for the mordenite and ferrierite after different cations sorption.

The sorption was conducted on monoionic form of mordenite and ferrierite (both zeolites are belonging to 5-1 structural group, and both has mor unit in the structure [7]). Figure $7 \mathrm{a}, \mathrm{b}$ presents some AAS results of heavy metal cations sorption as the function of the equilibrium metal ion concentration in the solution. Maximum cation sorption capacities of mordenite for particular ions are as follows: $\mathrm{Ag}^{+}-900, \quad \mathrm{Zn}^{2+}-365, \quad \mathrm{Cd}^{2+}-464, \quad \mathrm{~Pb}^{2+}-609$ and $\mathrm{Cr}^{3+}-202 \mathrm{mmol} / \mathrm{kg}$. And the same values for ferrierite: $\mathrm{Ag}^{+}-295, \mathrm{Zn}^{2+}-23, \mathrm{Cd}^{2+}-26, \mathrm{~Pb}^{2+}-167$ and $\mathrm{Cr}^{3+}-$ $31 \mathrm{mmol} / \mathrm{kg}$. Based on AAS results after sorption and desorption of cations, the proportion between ion-exchange and chemisorption in the sorption process of different heavy metal cations can be established. Figure $7 \mathrm{c}$, d shows the shares of sorption mechanisms on analyzed zeolites for metal cations studied, using the solution which initial 


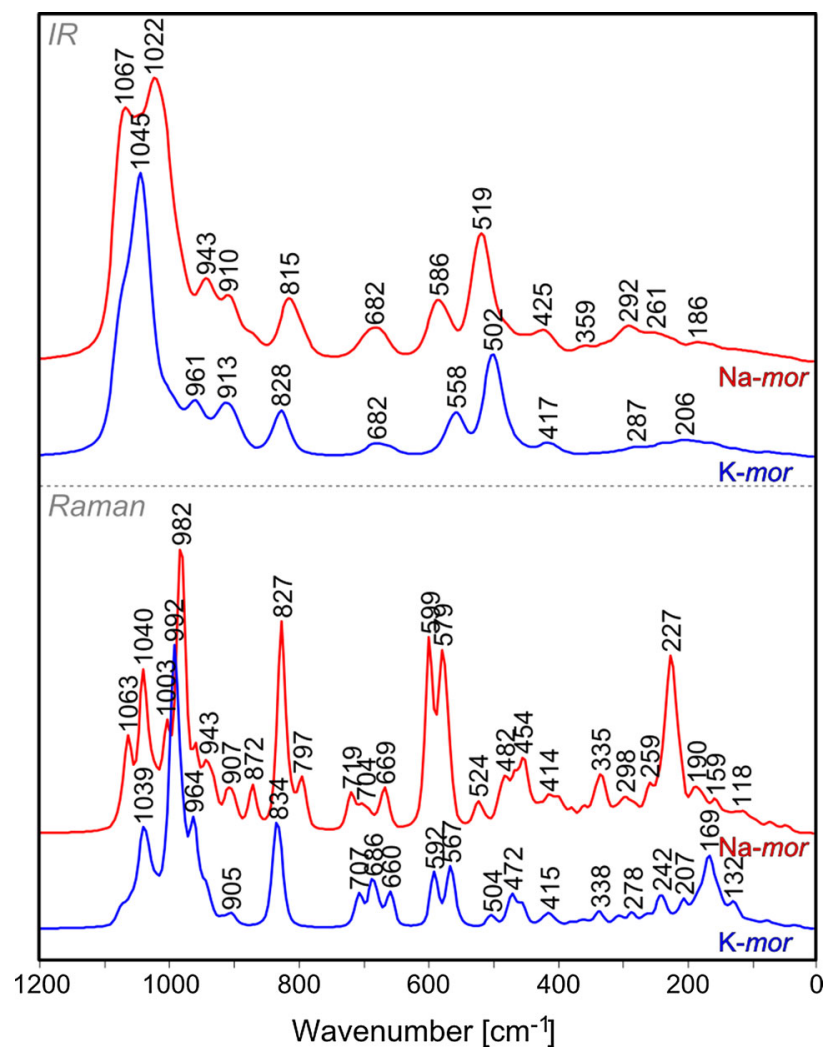

Fig. 6 Calculated IR and Raman spectra of mor model unit terminated by $\mathrm{Na}^{+}$and $\mathrm{K}^{+}$cations

concentration $5 \mathrm{mM} / \mathrm{dm}^{3}$. Sorption mechanisms and their contributions are influenced by the immobilized cation type and to a lesser extent by the zeolite structure. Ion-exchange dominates in the sorption process of divalent cations, while in the case of $\mathrm{Ag}^{+}$and $\mathrm{Cr}^{3+}$ ions, contribution of chemisorption is considerable. The amount of $\mathrm{Ag}^{+}$sorbed by both samples is distinctly higher than other cations. The small size may facilitate diffusion of this ions in zeolites framework, but it is also possible to precipitate the silver in the form of some sparingly soluble salts-this can this may explain the high proportion of chemisorption. Analysis of chromium ions $\left(\mathrm{Cr}^{3+}\right)$ sorption process on both zeolites proves that for trivalent cations chemisorption dominates over ion-exchange, however, as above, it is possible that some of these ions was precipitated as chromium hydroxide $\mathrm{Cr}(\mathrm{OH})_{3}$ [13]. Divalent ions are exchanged in comparable amounts-slight differences are due to different speciation in the aquatic environment [14], e.g. lead is present as $\mathrm{Pb}(\mathrm{OH})_{2}$, hence the largest sorption.

MIR spectra in the pseudolattice vibration range were collected in Fig. 8. It was found, that the band at about $560 \mathrm{~cm}^{-1}$ is sensitive to the ion exchange process and can be treated as some kind of indicator of sorption process. This observation well agrees with the results of calculations-this band is assign as connected with characteristic vibrations of mor unit. The second band in this region of the spectra, whose changes were observed, is at about $733 \mathrm{~cm}^{-1}$, although they are very negligible.

The most visible changes are observed in the FIR range (Fig. 9). This follows from the fact that in this range the bands due to cation vibrations appear. The magnitude of the changes in this range is dependent on the type of zeolite framework, type of cation (its ionic radii, charge, etc.) and its concentration in the initial solution [15]. In the case of mordenite, the FIR spectra of $\mathrm{Cd}$ - and $\mathrm{Pb}$-form are quite similar; with compared to spectrum of the Na-form, the intensities of bands at 167 and $96 \mathrm{~cm}^{-1}$ increase. Under the influence of $\mathrm{Na}^{+}$to $\mathrm{Ag}^{+}$exchange, the band at $112 \mathrm{~cm}^{-1}$ shift to $96 \mathrm{~cm}^{-1}$. In the case of $\mathrm{Cr}^{3+}$ ions the band at $112 \mathrm{~cm}^{-1}$ dominates. $\mathrm{Zn}^{2+}$ ions, in turn, cause a significant increase integral intensity in the whole analyzed range, which can be attributed to specific character of this ion or the precipitation of e.g. zinc oxide. In the case of ferrierite, dependences are similar.

It is well known that the FIR spectra of alkali and alkaline earth metal ion exchanged zeolites display wellresolved $\mathrm{Me}-\mathrm{O}$ vibration modes that are characteristic of their locations in the zeolite framework. Cationic positions are known only in a few zeolites with low $\mathrm{Si} / \mathrm{Al}$ ratio (e.g. zeolite Y $[9,16,17]$ or zeolite A $[18,19])$-with a high content of cations, therefore, in the case the other zeolites, the interpretation of results is difficult. However, based on the obtained results can be determined systematically change the intensity of the bands with increasing concentration of initial solution (Fig. 10). In the case of mordenite (Fig. 10a) ion exchange of sodium on lead cause clear systematically decrease in the intensity of the band at $112 \mathrm{~cm}^{-1}$. Smaller modifications are observed in the case of ferrierite (Fig. 10b), probably because they contain the least amount of cations, but also due to its dense structure. However, certain regularities can be observed-the increase in the number of $\mathrm{Cd}^{2+}$ ions in the structure increases the intensity of the band at about 195 and $113 \mathrm{~cm}^{-1}$ relative to the band at about $169 \mathrm{~cm}^{-1}$. The interpretation of this range of experimental spectra requires taking into account in theoretical analysis also the presence of long-range ordering, as well as the other than $\mathrm{Na}^{+}$and $\mathrm{K}^{+}$extra framework cations.

Ion-exchange properties of zeolites are connected with the presence of non-compensated negative charges and the presence of surface functional groups in crystalline lattice discontinuity points. This is a reversible process accompanied by the formation of weak bonds between the zeolite and metal ions (van der Waals bonds) [20]. Chemisorption process results the formation of stable inner-sphere complexes. This is due to the fact that functional groups of aluminosilicates network (mainly $\mathrm{OH}$ ) form strong chemical bonds with the ion without hydration layer [21]. In 
Fig. 7 Results of heavy metal cations removal on mordenite (a) and ferrierite (b) and contribution of ion-exchange and chemisorption mechanisms in the sorption process on mordenite (c) and ferrierite (d) respectively
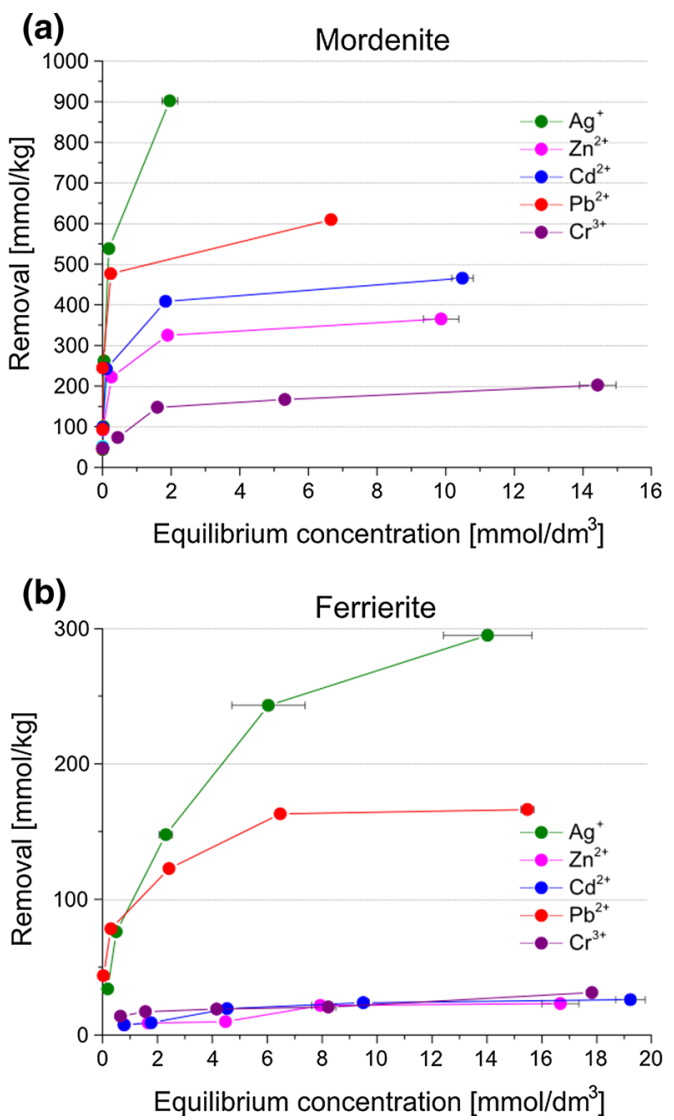

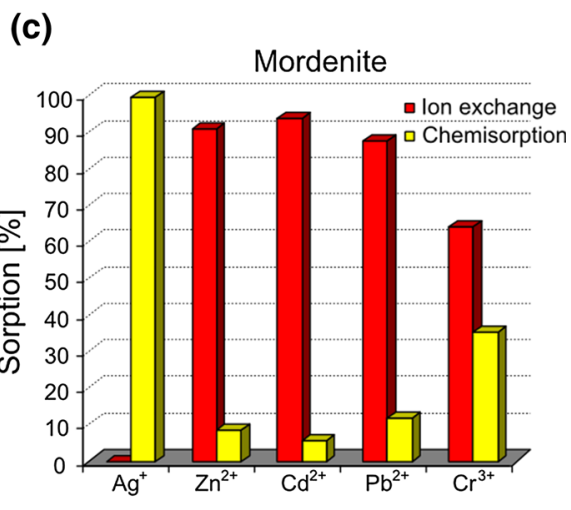

(d)

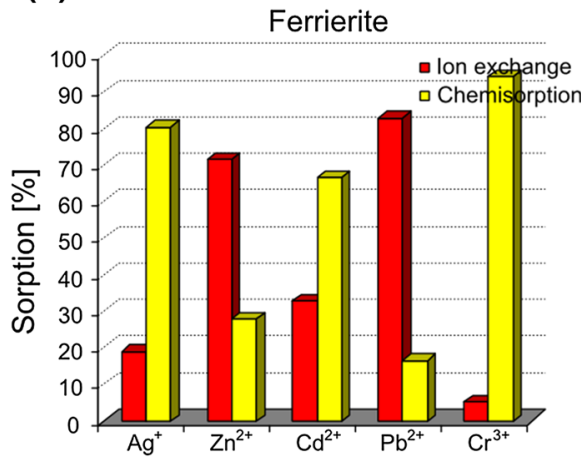

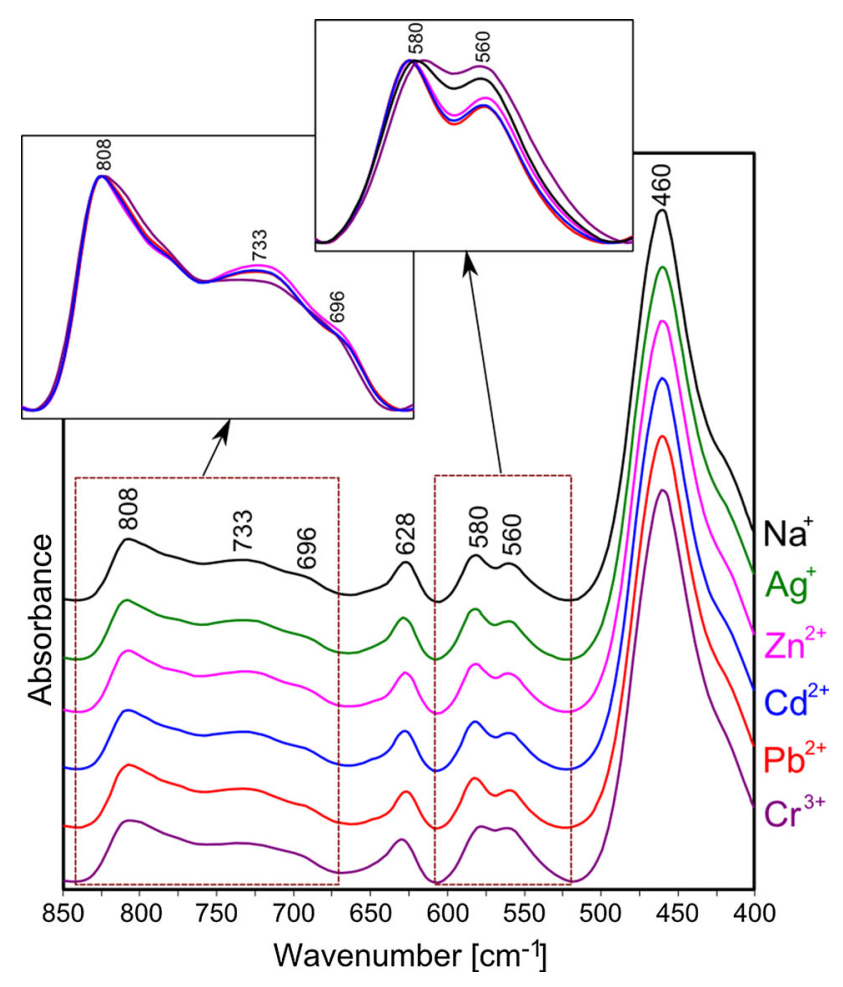

Fig. 8 MIR spectra of mordenite after sorption of heavy metal cations

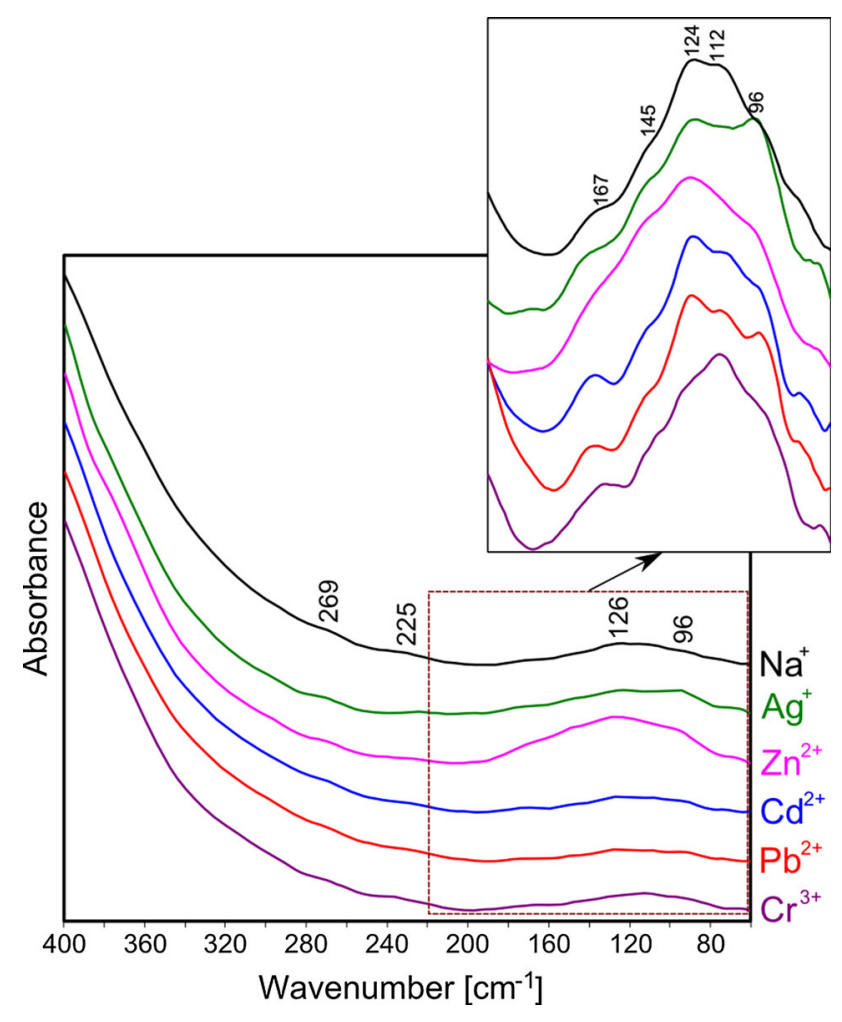

Fig. 9 FIR spectra of mordenite after sorption of heavy metal cations 

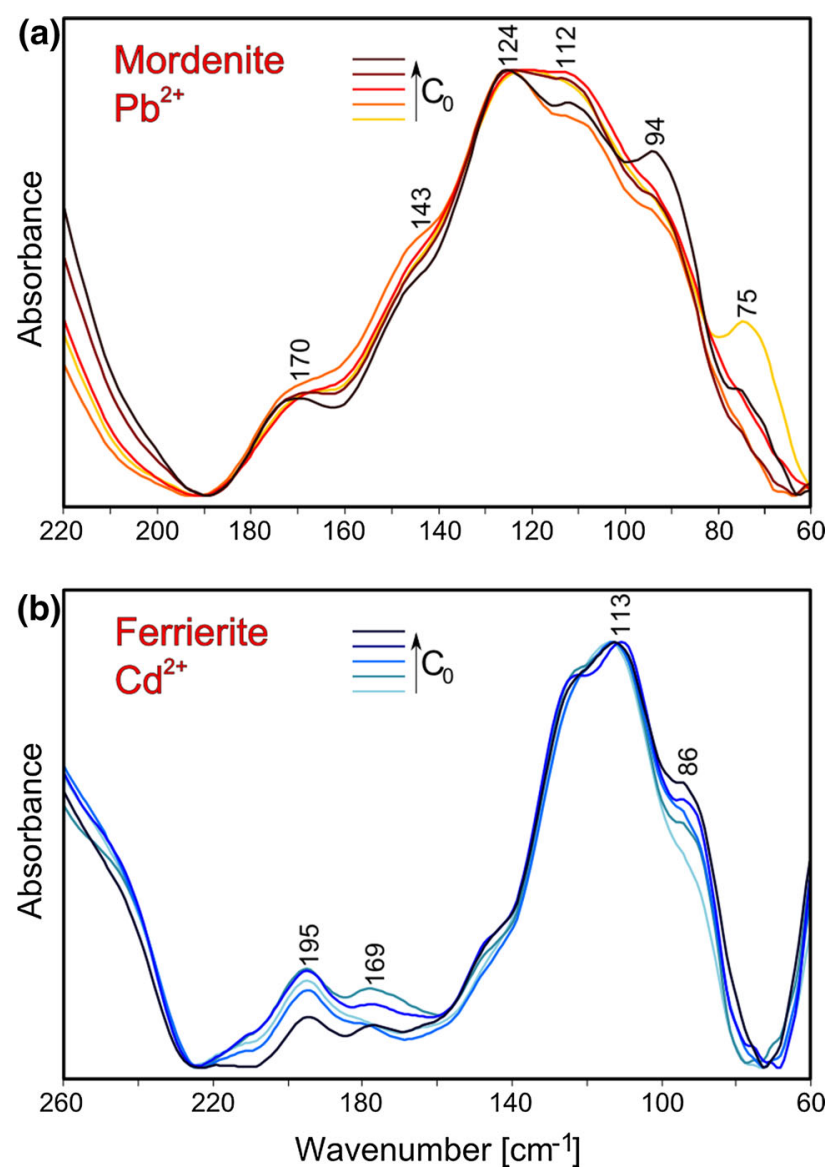

Fig. 10 FIR spectra of ferrierite (a) and mordenite (b) after heavy metal cations sorption at various concentrations of initial solution

zeolites the ion-exchange process usually dominates over chemisorption, however not in all cases. Zeolites used in this study have a high $\mathrm{Si} / \mathrm{Al}$ molar ratio (such as all pentasils). It follows from this a large share of chemisorption, which does not require negative charge like ion exchange and takes place in statistically random positions in the structure. Thus, changes in the spectra, especially in the MIR range, are unlikely. It is especially visible in the case of $\mathrm{Cr}^{3+}$ ions- these ions form the aqua complexes, which, due to its large size, have hindered diffusion in the skeleton (hence the low value of the sorption). Simultaneously at high $\mathrm{pH}$ (zeolites are characterized by high $\mathrm{pH}$ of surface), chromium tend to precipitate in the form of hydroxide. In the case chromium ions, no effect associated with the ionexchange process is visible in the MIR spectrum (Fig. 8) described in the manuscript band at 733 and $560 \mathrm{~cm}^{-1}$ not change. The situation is somewhat different in the case of silver ions, which do not hydrolyze in an aqueous environment and, therefore, their binding with the zeolite structure will be stronger. Confirmation can be found in the FIR spectrum, in which band at $96 \mathrm{~cm}^{-1}$ can be assigned to the $\mathrm{Ag}-\mathrm{O}$ vibrations (Fig. 9).

\section{Conclusions}

It has been found, that based on DFT calculations of vibrational spectra of small structural fragment (mor unit) models a characteristic vibrations can be identify and interpretation may be carried over to experimental spectrum.

MIR range spectra for zeolite measured after the sorption process show changes caused primarily by the ionexchange process. The presence of heavy metal cations in the zeolite structure causes the changes in the pseudolattice range of IR spectra. The bands, which are related to characteristic ring vibrations, become more intensive-in the case of mordenite, the band at about $560 \mathrm{~cm}^{-1}$ can be defined as a sorption indicator. Vibrational spectroscopy can be used in the studies of sorption properties.

An introduction of heavy metal cations into the structure of zeolites has caused the considerable modification of bands due to $\mathrm{Me}-\mathrm{O}$ vibrations in FIR spectra. Cation exchange in the structure of both analyzed zeolite structures cause changes the intensity and position of bands in the range $300-60 \mathrm{~cm}^{-1}$. In this case, systematic changes connected with the type of cation have been revealed, however this changes can not be fully interpreted, due to the nature of the research model.

Open Access This article is distributed under the terms of the Creative Commons Attribution 4.0 International License (http://crea tivecommons.org/licenses/by/4.0/), which permits unrestricted use, distribution, and reproduction in any medium, provided you give appropriate credit to the original author(s) and the source, provide a link to the Creative Commons license, and indicate if changes were made.

\section{References}

1. E.A. Jenne, Adsorption models, in Adsorption of Metals by Geomedia: Variables Mechanism and Model Applications, ed. by E.A. Jenne (Academic, San Diego, 1998)

2. M. Król, W. Mozgawa, W. Jastrzębski, K. Barczyk, Micropor. Mesopor. Mater. 156, 181-188 (2012)

3. W. Mozgawa, M. Król, T. Bajda, J. Mol. Struct. 924-926, 427-433 (2009)

4. F. Pechar, D. Rykl, Can. Mineral. 21, 689-695 (1983)

5. H. Mikosch, E.L. Uzunova, G. St, Nikolov. J. Phys. Chem. B 108, 13200-13207 (2004)

6. D.W. Breck, Zeolite Molecular Sieves (Wiley, London, 1974)

7. Ch. Baerlocher, L.B. McCusker, Database of Zeolite Structures. http://www.izastructure.org/databases/

8. W. Mozgawa, T. Bajda, J. Mol. Struct. 792-793, 170-175 (2006)

9. M.D. Baker, J. Godber, G.A. Ozin, J. Am. Chem. Soc. 107, 3033-3043 (1985)

10. E.M. Flaningen, H. Khatami, H.A. Szymański, Adv. Chem. Ser. 101, 201 (1971)

11. F. Pechar, D. Rykl, Infrared Spectra of Natural Zeolites, Rozpr. Ceskosl. Akad. Ved. (Praha, 1985) 
12. E.L. Uznanova, GSt Niklov, J. Phys. Chem. B 104, 7299-7305 (2000)

13. H.B. Bradl, J. Colloid Interface Sci. 277, 1-18 (2004)

14. M.S. Berber-Mendoza, R. Leyva-Ramos, P. Alonso-Davila, J. Mendoza-Barron, P.E. Diaz-Flores, J. Chem. Technol. Biotechnol. 81, 966-973 (2006)

15. M. Król, W. Mozgawa, K. Barczyk, T. Bajda, M. Kozanecki, J. Appl. Spectr. 80(5), 662-668 (2013)

16. H. Esemann, H. Förster, J. Chem. Soc. Chem. Commun. 1319-1320 (1994). doi:10.1039/C39940001319

17. I.A. Brodskii, S.P. Zhdanov, A.E. Stanevich, Opt. Spektrosk. 30, 58-62 (1971)
18. M.D. Baker, J. Godber, K. Helwig, G.A. Ozin, J. Phys. Chem. 92, 6017-6024 (1988)

19. K.S. Smirnov, M. Maire, C. Brémard, D. Bougeard, Chem. Phys. 179, 445-454 (1994)

20. V.J. Inglezakis, M.D. Loizidou, H.P. Grigoropoulou, Water Res. 36, 2784-2792 (2002)

21. A. Godelitsas, Transition metal complexes supported on natural zeolitic materials: an overwiew, in Natural Microporous Materials in Environmental Technology, ed. by P. Misaelides, et al. (Kluwer, Dordrecht, 1999) 